\title{
Designing an advanced electrode of mixed carbon materials layered on polypyrrole/reduced graphene oxide for high specific energy supercapacitor
}

\begin{abstract}
A free-standing and flexible film is fabricated by layering multiwalled carbon nanotube/reduced graphene oxide/nanocrystalline cellulose composite on polypyrrole/reduced graphene oxide composite layer. The bilayer composite film is prepared by in-situ polymerization through a vacuum filtration method followed by a chemical reduction in the presence of hydrazine vapor and used as an electrode material for supercapacitor. The aggregation of multiwalled carbon nanotube and graphene oxide in the composite are addressed effectively by the support of nanocrystalline cellulose that favors ions movements in the composite. The symmetrical supercapacitor device developed in this study combines the features of pseudocapacitor and electrical double layer capacitor and delivers outstanding supercapacitive properties. As manifested by the electrochemical results, the device exhibits a high specific capacitance of $882.2 \mathrm{Fg}-1$, remarkable cycling stability of $\sim 90 \%$ over 10,000 cycles, and high specific energy of $44.6 \mathrm{Wh} \mathrm{kg}-1$ with a high specific power of $2889.9 \mathrm{~W} \mathrm{~kg}-1$, which outperformed many other reported polypyrrole-based materials for supercapacitors. This makes the bilayer a promising candidate for future high performance energy storage devices.
\end{abstract}

Keyword: Supercapacitor; Layer-by-layer; Multiwalled carbon nanotube; Graphene oxide; Nanocrystalline cellulose; Polypyrrole 